\title{
Families of fragmenting granules and their relation to meso- and supergranular flow fields
}

\author{
Th. Roudier ${ }^{1}$, F. Lignières ${ }^{2}$, M. Rieutord ${ }^{2,3}$, P. N. Brandt ${ }^{4}$, and J. M. Malherbe ${ }^{5}$ \\ ${ }^{1}$ Laboratoire d'Astrophysique, Observatoire Midi-Pyrénées, 57 avenue d'Azereix, BP 826, 65008 Tarbes, France \\ ${ }^{2}$ Laboratoire d'Astrophysique, Observatoire Midi-Pyrénées, 14 avenue E. Belin, 31400 Toulouse, France \\ 3 Institut Universitaire de France, France \\ ${ }^{4}$ Kiepenheuer-Institut für Sonnenphysik, Schöneckstrasse 6, 79104 Freiburg, Germany \\ 5 LESIA, Observatoire de Paris, Section de Meudon, 92195 Meudon, France
}

Received 17 February 2003 / Accepted 10 June 2003

\begin{abstract}
D analysis $(x, y, t)$ of the granular intensity field (11-hour time sequence from the Swedish Vacuum Solar Telescope on La Palma, Canary Islands), demonstrates that a significant fraction of the granules in the photosphere are organized in the form of "Trees of Fragmenting Granules" (TFGs). A TFG consists of a family of repeatedly splitting granules, originating from a single granule at its beginning. A striking result is that TFGs can live much longer (up to $8 \mathrm{~h}$ ) than individual granules (10 min). We find that $62 \%$ of the area covered by granules belongs to TFGs of a lifetime $>1.5 \mathrm{~h}$. When averaged in time, such long-lived TFGs correspond to coherent diverging flows which may be identified as mesogranules. We also find a correlation between the network and the spatial distribution of TFGs.
\end{abstract}

Key words. Sun: photosphere - Sun: granulation

\section{Introduction}

A knowledge of the physical nature of the different flow scales on the Sun is necessary to envisage a realistic model of the generation (production) and diffusion of the magnetic flux on the surface of the quiet Sun, in particular to build the network in the quiet regions, or to understand the action of the different flow scales in the destruction of sunspots. More precisely, the processes which create, and then fragment and disperse Ephemeral Regions populating the quiet network, is not well known today and remains quite speculative (Hagenaar et al. 2003). The generation of these bipolar regions by the local action of the turbulent convection (small-scale local dynamo) is also invoked to explain the low sensitivity of these small dipoles to the sunspot cycle (Hagenaar et al. 2003). The mechanisms produced by the turbulent convective motions at the different scales have to be elucidated to determine in more detail the role of the turbulent dynamo in forming these dipoles.

The links between the various scale motions on the Sun's surface are still poorly understood today. Recently the physical nature of the largest flow scales, i.e. the meso- and supergranulation, has been found to vary depending on the spatial and temporal windows used to compute the flow fields (see Rieutord et al. 2000).

Send offprint requests to: Th. Roudier, e-mail: roudier@bagn.obs-mip.fr
On the one hand, low resolution measurements of the properties of solar photospheric flows support the existence of different convective scales. In particular, the work of Muller et al. (1992) and Shine et al. (2000) shows the persistence of the supergranulation, and the motion of the mesogranules toward the supergranular boundaries in accordance with the model of Simon et al. (1991). On the other hand, Rieutord et al. (2000) found that computing the flow fields with high spatial $\left(0.7^{\prime \prime}\right)$ and temporal resolution $(5 \mathrm{~min})$ reveals the important role of the strong positive divergences (SPDs) in forming the larger scales (meso- and supergranulation).

To link the measurements at low and high spatial resolution, one needs a time sequence of the solar granulation observed over several hours with a field of view at least $1^{\prime} \times 1^{\prime}$, to get reliable results up to the largest scale, i.e. the supergranulation. Here we make use of an exceptional $11 \mathrm{~h}$ time sequence obtained at the SVST at La Palma.

Using this time sequence we try to understand the different results from low and high resolution analyses, as well as to determine the links between the three scales: granulation, mesogranulation, and supergranulation. Granule evolution was investigated by many authors (e.g. Spruit et al. 1990) in order to characterize the dynamics of the Sun's surface.

One of these approaches has been developed by Kawaguchi (1980) using a 46-min time sequence; he showed the existence of long-lived structures called "families of active granules". From his 2D numerical model Ploner (1994) concluded that 
fragmenting granules can split repeatedly for several generations and discussed the link between these long-lived structures and the mesogranulation. Using time-slice of the Sun surface, Müller et al. (2001), found that fragmenting granules can survive by means of their descendants for more than $3 \mathrm{~h}$. Their works suggest that mesogranular flow fields may be generated by actives granules.

In this paper we first present and discuss a new 3D automatic time labeling method of the solar granules. The application of this method to the $11 \mathrm{~h}$ time sequence obtained at the SVST confirms the existence of families of active granules and allows a detailed analysis of their statistical properties. We discuss the link between these long-lived families and the mesogranules. Finally, we show some properties of these long-lived structures with relation to the network.

\section{Data}

Our analysis is based on a series of 1500 CCD images of size $512 \times 512$ pixels $\left(64^{\prime \prime} \times 64^{\prime \prime}\right)$ of the solar granulation obtained under excellent seeing conditions with the $50 \mathrm{~cm}$ Swedish Vacuum Solar Telescope on La Palma (Canary Islands) on June 5, 1993.

A quiet Sun section of that series is extracted from a longer set of observations obtained from 08:07 UT to 19:07 UT close to a pore in spot group NOAA 7519 at position N05, E15. The duration of the analyzed sequence is $8.75 \mathrm{~h}$ with a mean time step of $21 \mathrm{~s}$. The images were corrected for dark current and gain, rigidly aligned, and destretched. They have been filtered to remove five-minute oscillations. Most details of the image reduction procedure are given in Simon et al. (1994).

\section{Analysis method}

In this section we present the method used to detect families of splitting granules.

The transformation of the intensity map into a binary map has been performed following the criterion defined by Strous (1994), i.e. the local curvature of the intensity field $a=I_{1}-$ $2 I_{2}+I_{3}\left(I_{1}, I_{2}, I_{3}\right.$ being the intensity of three adjacent pixels) computed in four directions. When the second spatial derivatives have the same sign in the four directions, for example negative, the pixel belongs to a bright object (granule), in the opposite case it belongs to a dark intergranule. Before this segmentation, we convolve each frame with a Gaussian window, the size of which is adjusted such as to avoid over- or undersegmentation. In order to avoid false identifications of granules and keep the granule core whatever their size, we used a Gaussian window of 6 pixels size $\left(0.75^{\prime \prime}\right)$.

The segmented granules shown in Fig. 1 have relatively large distances from each other. The granule radial expansion velocity being of order of $2 \mathrm{~km} \mathrm{~s}^{-1}$ and their velocity smaller than $1.0 \mathrm{~km} \mathrm{~s}^{-1}$, such a segmentation prevents spurious granule mixing as granules move less than one pixel between consecutive frames at a lag of $21 \mathrm{~s}$.

Once the segmentation is performed, objects are labeled in each frame. Then, we follow the evolution of the granules by identifying the temporal links in the $3 \mathrm{D}$ data box $(2 \mathrm{D}$ in
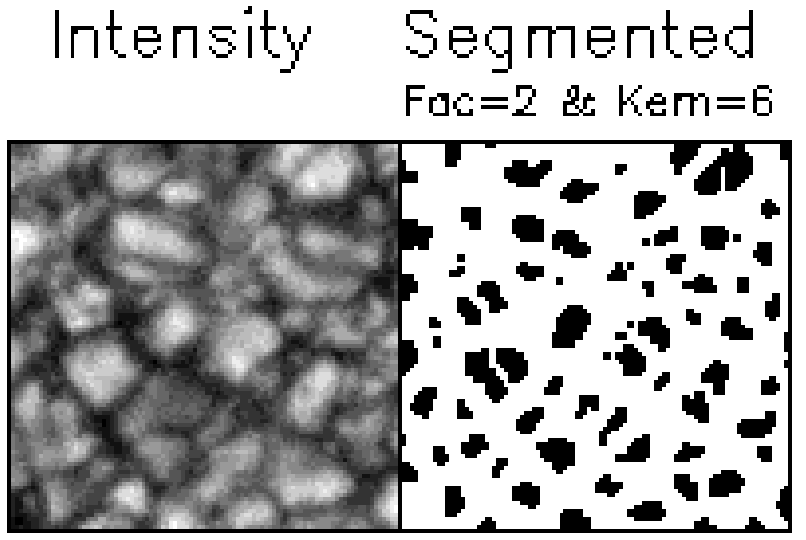

Fig. 1. Example of intensity and binary map using a Gaussian window of kern $=6$ pixels size $\left(0.75^{\prime \prime}\right)$ and fac $=2$ pixels size $\left(0.25^{\prime \prime}\right)$ for the structuring element of the "Opening" operator.

space and 1D in time). This step is complicated because granules change shape during their lifetime and it is well known that it is quite difficult to define the life history of a typical granule (Spruit et al. 1990). Hence, splitting into multiple objects, merging of objects, and disappearances or appearances between two successive images have all to be taken into account. As our segmentation keeps the core of all the granules, we avoid erroneous detections of temporal links between adjacent granules due to the different sources of noise or the segmentation technique. Two criteria were used to perform the temporal labeling of the granules:

1. From a frame at time $t$ to the next at time $t+1$, we detect for each granule identified at the time $t$ if there exists a granule at the time $t+1$ at the same location with a minimal common area. As our segmentation was very restrictive, we chose the minimal common area to be one pixel;

2. When two or more granules merge, we kept only the label of the larger one in the area.

When these criteria are met, objects are labeled from one frame to the next with the same number, otherwise the number is changed to a new one. We constructed in this way $97053 \mathrm{ob}-$ jects contained within the 3D $(x, y, t)$ box. Of these 54135 had a lifetime greater than one frame. And of those 54135 objects, 5549 had at least one splitting and formed the set of what we shall call "Trees of Fragmenting Granules" (TFGs). The remaining 48586 objects are called individual granules. TFGs are families of repeatedly splitting granules, originating from a single granule at its beginning. Exploding granules represent the most vigorous case of fragmenting granules. Figure 2 shows a $3 \mathrm{D}$ view and a time slice of the longest lived TFG. Horizontal arrows indicate the splittings visible in the time slice. This shows that, with 2D time slices, the temporal continuity is only visible during a fraction of the TFG lifetime. Because granules split in any directions, the temporal connection of the repeatedly splitting granules is completely described only in the 3D view.

The method to detect the TFGs depends on the segmentation and on the criterion chosen to control granule merging. The sensitivity of the method to changes of the segmentation 


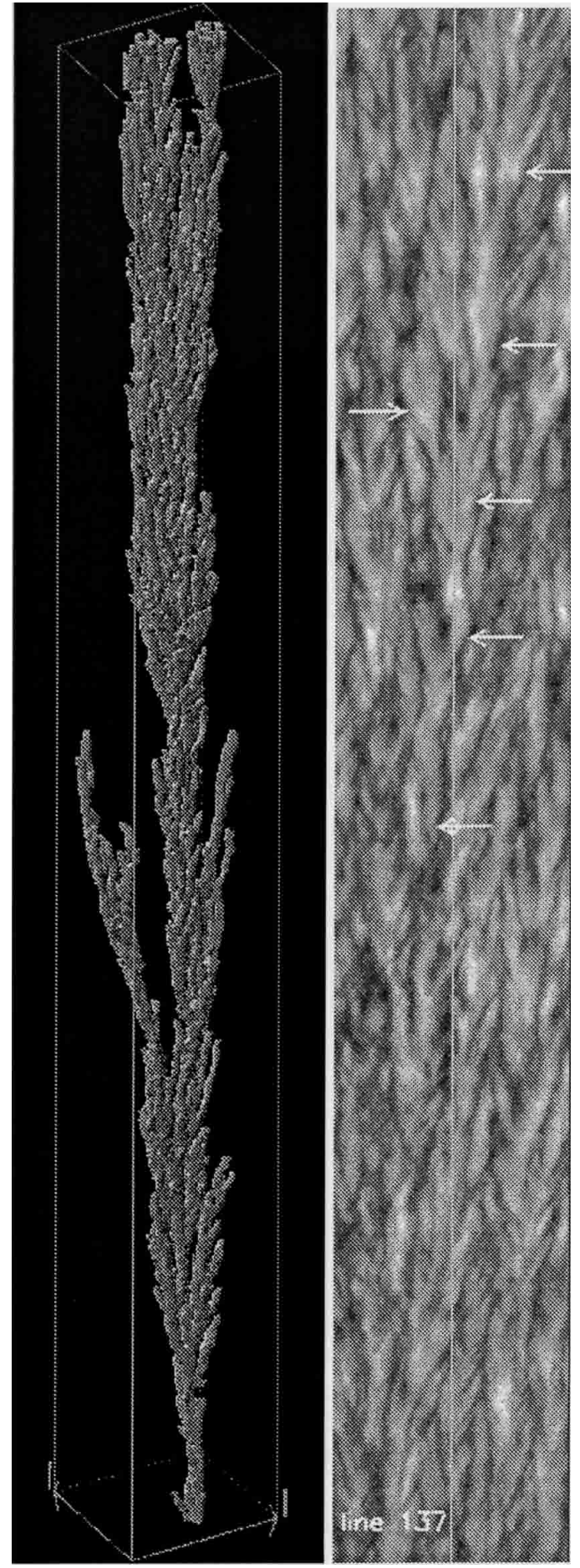

Fig. 2. Left: 3D $(x, y, t)$ representation of the longest-lived TFG $(8 \mathrm{~h} 10 \mathrm{~min})$ of our time sequence which influenced a total area of $15^{\prime \prime} \times 17^{\prime \prime}$ during its life. Right: $2 \mathrm{D}$ time slice of one part of the TFG where the repeatedly connected splitting granules, indicated by horizontal arrows, are clearly visible.

parameters, i.e. the Gaussian window or the size of the structuring element, has been tested. We found the method to be quite robust with respect to the temporal labeling of granules in the range of window sizes of 5-8 pixels and of structuring elements of 2-3 pixels. Outside this range the granules are overor under-segmented leading to a non-conform labeling when compared with direct labeling by eye from the intensity field.

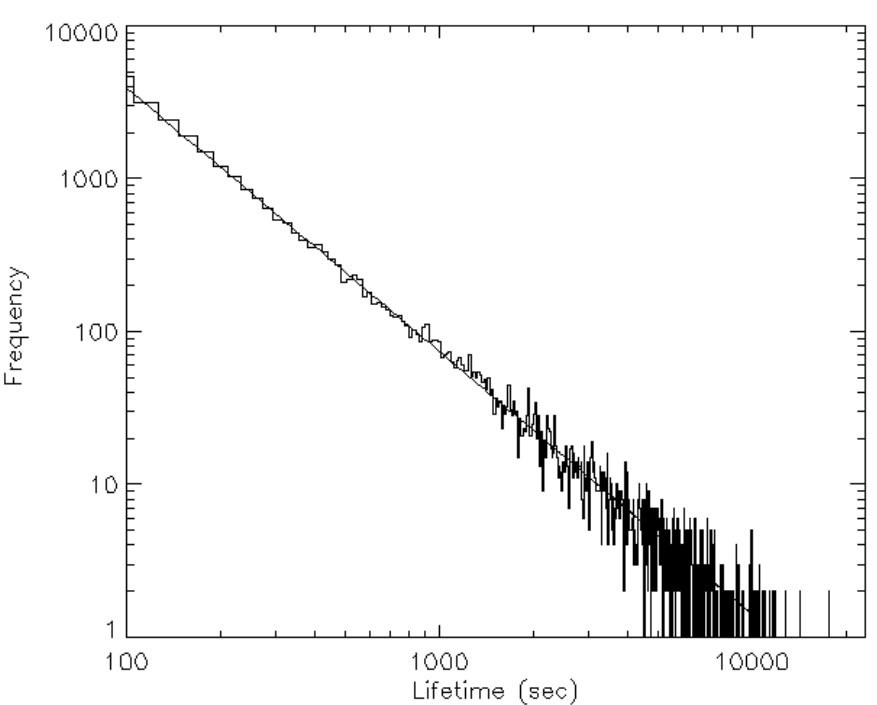

Fig. 3. Lifetime histogram of individual granules and TFGs. It is well fitted by a power law with an exponent equal to -1.72 .

The increase of the minimal common area does not modify the results described here because only small granules are affected and these correspond in general to the end of the life of the granules. Finally, the segmentation parameters were also successful in reproducing the statistical properties of the granule lifetimes published by Title et al. (1989) (see Fig. 4 described in the next section).

We tested also the sensitivity of the TFG detection to the granule merging criterion by considering another criterion. We kept the number of the older of the two granules which merge at time $t-1$ as the label of the granule at time $t$. We verified that this change did not affect the statistical results (like the lifetime histograms, e.g. Fig. 3) found previously when keeping the label of the larger granule. This can be explained in the following way.

The number of merging granules relatively to the total number of granules is very low, i.e. $6.6 \times 10^{-3}$; this is due to our very strict segmentation method which reduces very strongly the merging. Moreover, the number of families that undergo a change of the family number is very low, i.e. $9 \times 10^{-5}$ yielding around 30 families, with respect to the total number of families (or granules labeled in time). When looking into more detail, most of the merging cases $(97 \%)$ were found not to affect the family number because the larger granules correspond to the older ones, or the merging granules belong to the same family at time $t-1$. Half of the remaining $3 \%$ of merging granules are found to be produced by granules whose lifetimes, at time $t$, are smaller than 5 min.

Hence, the time labeling of the longest-lived families is only affected at their beginning which modifies their origins but not very much their lifetimes.

Regarding the remaining $1.5 \%$, the merging affects the temporal labeling of individual granules and TFGs but their number is very small compared to the total number of families detected in the data cube. We note for these families some trends to collect smaller granules because of the choice of the criterion "number of older granule when merging" is not a local 


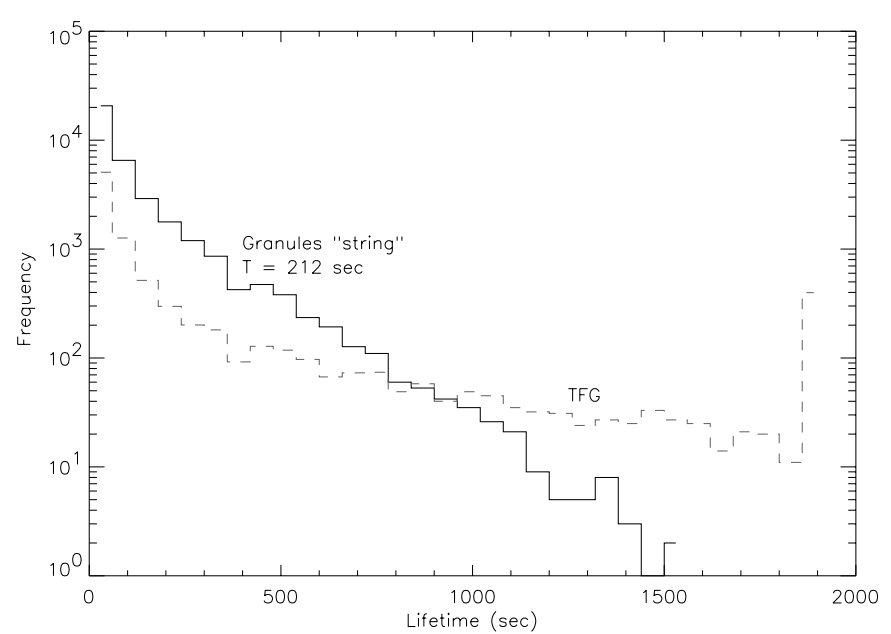

Fig. 4. Lifetime histograms (solid line) of granules for a set of $30 \mathrm{~min}$ of our time sequence (i.e. the same duration as the SOUP sequence) measured by the "string" method (like Title et al. 1989) and by our method (dashed line).

criterion. This is why, in our opinion, "the number of the larger when merging" for time labeling is more suitable because it is a local criterion. It avoids the artificial growth of families developing in this way. However, our analysis shows the very small effects of this choice on the statistical analysis.

\section{Families of long-lived, repeatedly fragmenting granules: The TFGs}

In this section, we present the statistical properties of the 5549 TFGs found in our sample. We characterize their lifetimes, their expansion radius and velocity, the area covered by TFGs as well as the number of splittings undergone during their lifetime.

As mentioned previously, TFGs are related to the families described by Kawaguchi (1980) in his 46-min time sequence. By comparison, the most striking result of our analysis is the existence of long-lived families, with the longest one lasting 8 h 10 min (shown in Fig. 2). This clearly appears in Fig. 3 which shows the histogram of the lifetimes of individual granules and TFGs. Individual granules have been added to complete the statistics at small lifetimes.

This lifetime histogram is well fitted by a power law $N \propto t^{\gamma}$, with $\gamma=-1.72$. Such a self-similar behavior indicates that we cannot define a characteristic lifetime of the TFGs. By contrast, previous works on the lifetime of solar granulation defined a characteristic life time as the decay rate of an exponential law which fitted well the histogram of individual granule lifetime. The difference between these results and the power law found here is clearly due to the definition of the time labeling. In our case, we detect structures which can merge or split while conserving their temporal continuity. In contrast to this, in the SOUP lifetime measurements for instance, the granules were only considered between their birth and a splitting or a merging event; this was called the "string" method.

Figure 4 exhibits the comparison, for a set of $30 \mathrm{~min}$ of our time sequence (i.e. the same duration as the SOUP sequence),

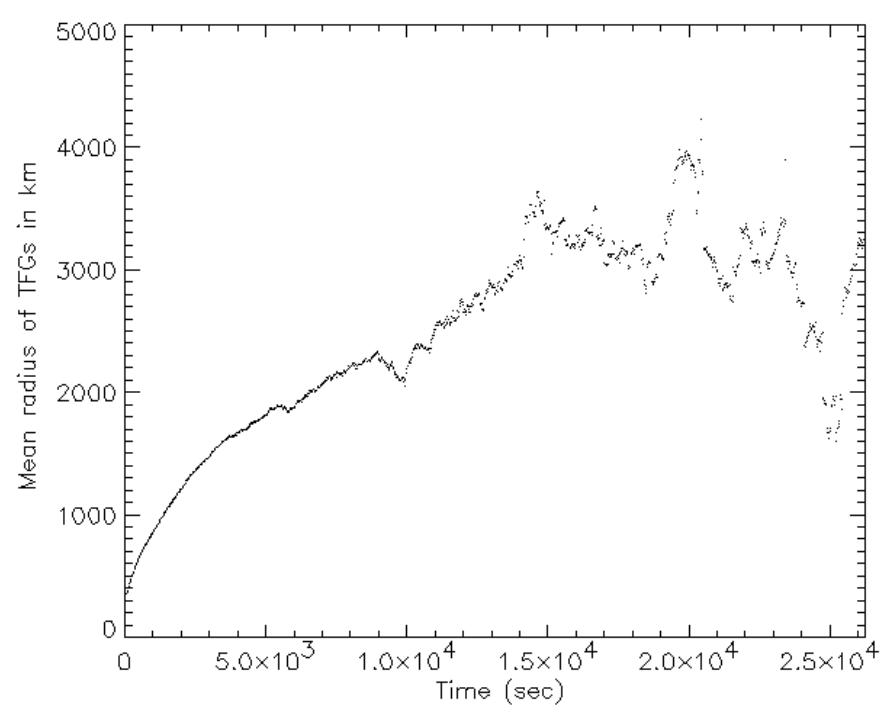

Fig. 5. Average radius of the TFGs as a function of time.

between the histogram of granule lifetimes measured by the string method (we find an exponential law with $T=212 \mathrm{~s}$ in accordance with the SOUP results presented in Title et al. 1989) and the histogram of the granules and TFGs measured by our method.

We now consider the spatial expansion of TFGs which results from the collective effects of granule splittings. The radius of TFGs, defined as the distance of the most distant granule from the centre of gravity of the TFG at that time, has been computed at each time step during the lifetime of all 5549 TFGs. A mean radius, averaged over all TFGs, is then calculated and Fig. 5 exhibits its time evolution. The figure shows that the mean radius increases up to $3000 \mathrm{~km}\left(4.1^{\prime \prime}\right)$ in $15000 \mathrm{~s}(4.16 \mathrm{~h})$; after this the mean radius stays more or less constant up to $23500 \mathrm{~s}(6.5 \mathrm{~h})$. The maximum mean radius of $3200 \mathrm{~km}$ corresponds to the typical radius of a mesogranule.

The expansion velocity derived from the mean radius evolution shows a maximum of $0.85 \mathrm{~km} \mathrm{~s}^{-1}$ after $200 \mathrm{~s}$, cf. Fig. 6 . This initial step is mainly due to the proper granule expansion. Then the velocity decreases continuously during one hour until it becomes statistically stationary around $0.15 \mathrm{~km} \mathrm{~s}^{-1}$. Beyond $10000 \mathrm{~s}$, the expansion velocity shows very large fluctuations (not shown here) associated to the mean radius fluctuations shown in Fig. 5. They are due to the relatively small number of TFGs in this range of lifetime.

The quasi monotonous expansion of TFGs is reflected in the area they cover at the end of their lifetime. Figure 7 shows that the final area covered by TFGs increases as a function of their lifetimes, the plot being well fitted by a power law Area $\propto$ $t^{\alpha}$, with $\alpha=5 / 4$. The figure also shows that most of the TFGs cover an area smaller than a disk of radius 4 " which is the mean radius of a mesogranule.

Related to the expansion and the lifetime of TFGs is the total number of splittings that occur in each TFG. As shown in Fig. 9, this number increases with the lifetime of TFGs the relation being fitted by a power law $N \propto t^{\beta}$, with $\beta=1.8$.

Although the number of TFGs decreases with lifetime, long-lived TFGs still cover a very significant proportion of the 


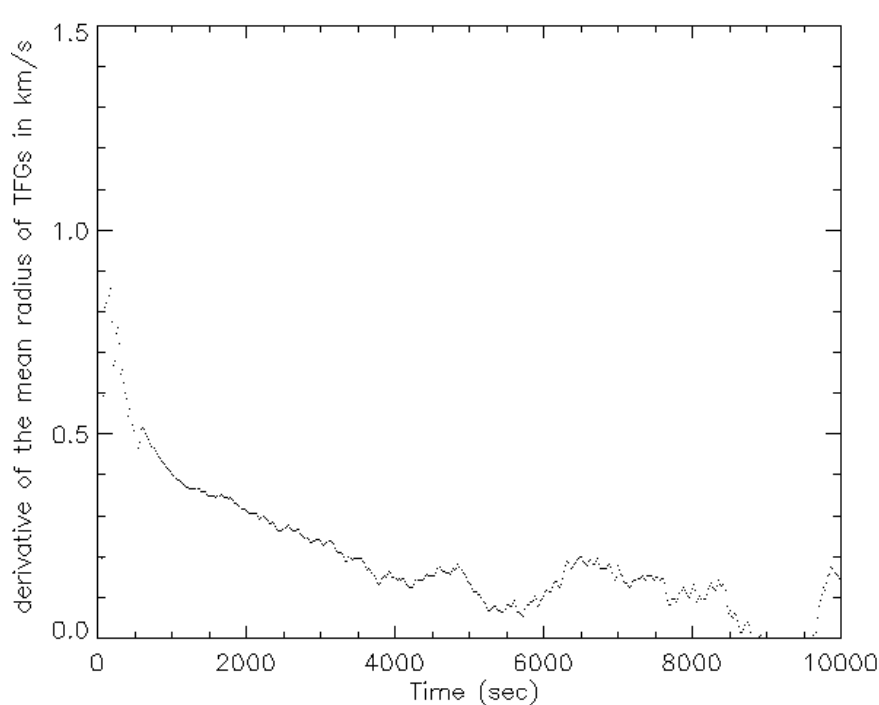

Fig. 6. Expansion velocity of the TFGs as a function of time.

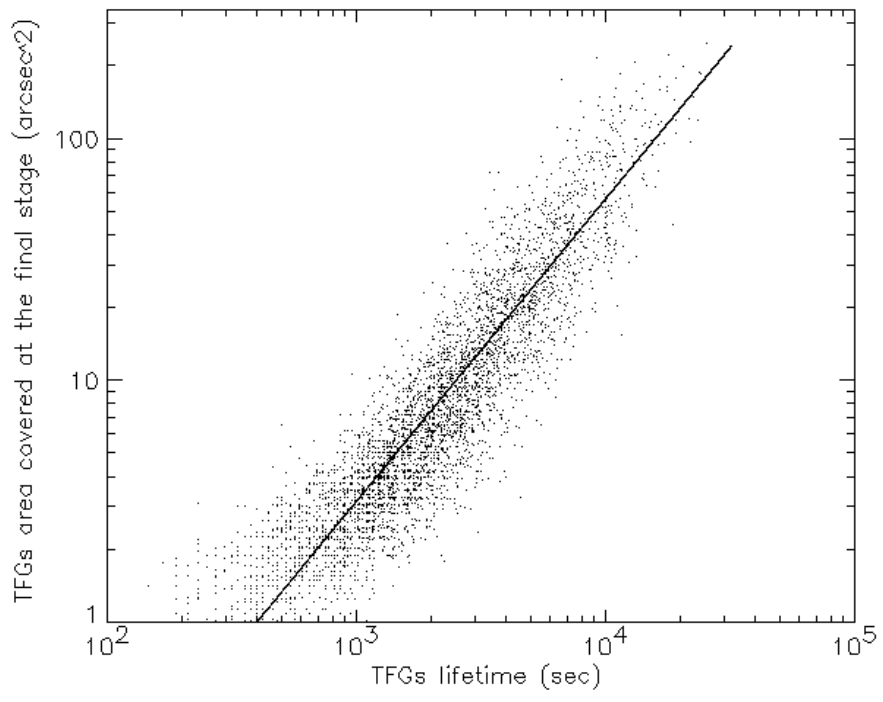

Fig. 7. Area covered by TFGs as a function of their lifetimes.

solar surface. This is shown in Fig. 8 where the proportion of the total area covered by granules belonging to TFGs living more than $1.5 \mathrm{~h}$ is displayed during the whole sequence. We see that the area contribution steeply increases in the first hour of the sequence; it then stabilizes to a platform slightly above $15 \%$ up to one hour before the end. The lack of contribution at the beginning and the end of the sequence is due to the loss of the TFGs lasting more than $1.5 \mathrm{~h}$ which are cut by the begin and end of that period. Such a platform shows that long-lived families are permanently present on the Sun's surface. Moreover, from the total area covered by all granules, i.e. $24 \%$, with our segmentation, we found that $62 \%$ of the granule area belongs to TFGs of a lifetime greater than $1.5 \mathrm{~h}$.

The large area covered as well as the temporal coherence of long-lived TFGs suggest that they can play a major role in the dynamics of the solar convection. In the next section, we shall investigate the contribution of these coherent structures to the flow field and relate it to mesogranulation.

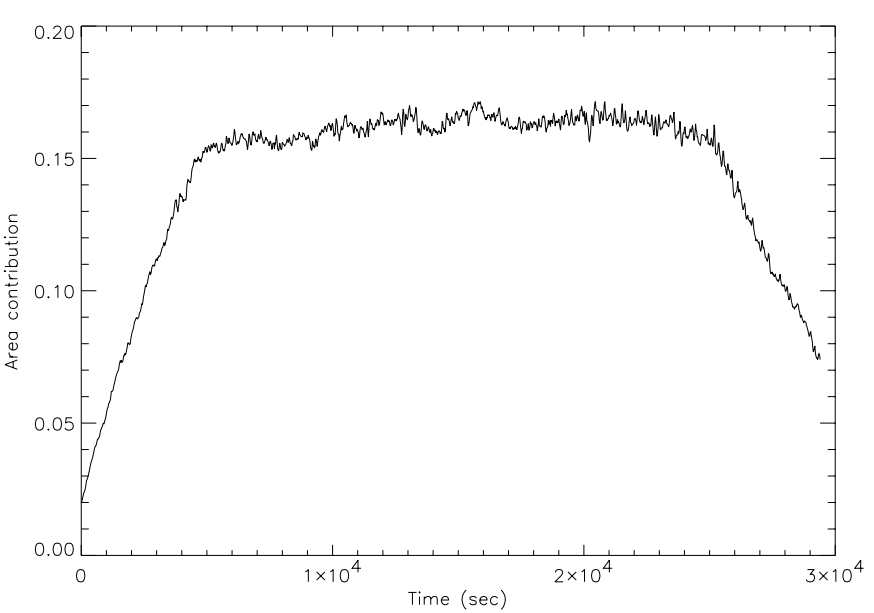

Fig. 8. Time evolution of the area of granules belonging to TFGs living $>1.5 \mathrm{~h}$ normalized to the total granule area.

\section{Relation between TFGs and mesogranules}

In the previous section, we showed that long-lived TFGs share some properties with the classical mesogranules, namely the size and the lifetime. In this section, we shall specify this relation by computing the time averaged velocity field at the location of long-lived TFGs.

Flow fields of various TFGs have been obtained using different temporal windows but the same spatial window of $1.5^{\prime \prime}$, a value which is commonly used in the literature. The first temporal window used for each family is its full lifetime. The mean flow fields of the three TFGs shown in Fig. 2 and the one shown in Fig. 10 are plotted in Fig. 11. The mean flow fields of Fig. 11 were computed at the locations where TFGs are present. Only granules belonging to each family were used to derive the velocities in order to delimit the region of the TFG properly. Similar flow fields are obtained if all the granules located in the same field are used.

As can be seen in Fig. 11, the flow fields show a more or less symmetric diverging aspect at the scale of mesogranules. In other words, one can say that the mean flow of these longlived TFGs is equivalent to a diverging flow at the mesoscale, that is to mesogranules according to the definition given by various authors in the literature (e.g. Simon et al. 1991).

Further evidence of the influence of long-lived TFGs on the large-scale dynamics and its links with mesogranules is given by Figs. 12 and 13. Both figures show the flow field averaged over the whole time sequence ( $8 \mathrm{~h} 45 \mathrm{~min}$ ) but Fig. 13 also displays the projection of the longest-lived TFG shown in Fig. 10. We observe that the granules belonging to that TFG are concentrated at the location where the mean flow field shows a diverging flow at the mesoscale.

In order to investigate the evolution of the TFG flow fields during their lifetime, different temporal windows were used in the computation, i.e. $15 \mathrm{~min}, 30 \mathrm{~min}$, and $60 \mathrm{~min}$, with a constant spatial window of $1.5^{\prime \prime}$. An example of the resulting flow fields is shown in Fig. 14. The main conclusion: is by increasing the temporal and spatial window the flow fields of a TFG appear as a nearly symmetric divergent velocity field at 


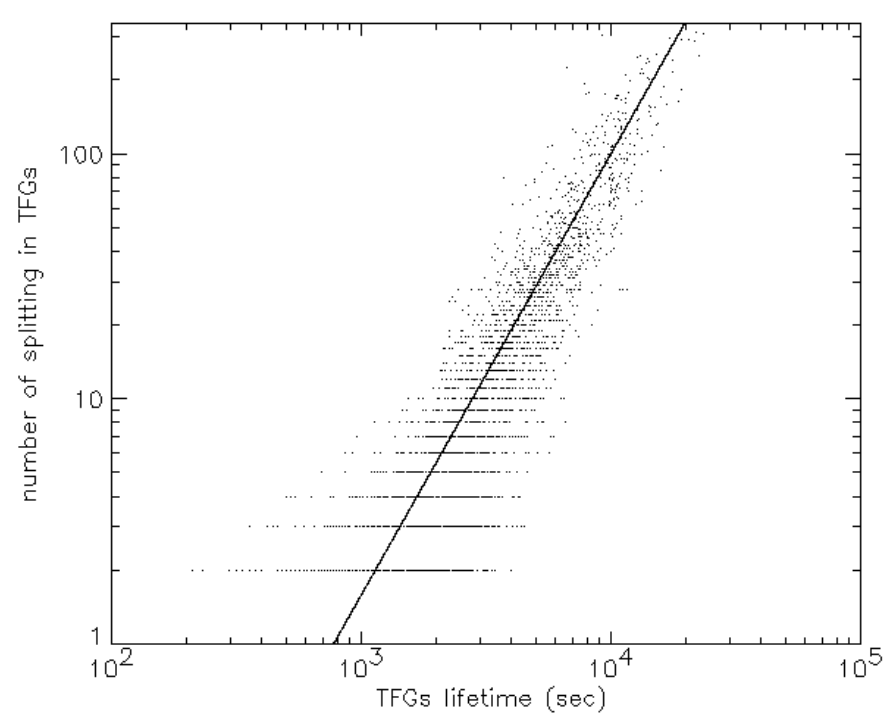

Fig. 9. Number of granule splittings in TFGs as a function of the TFG lifetimes.
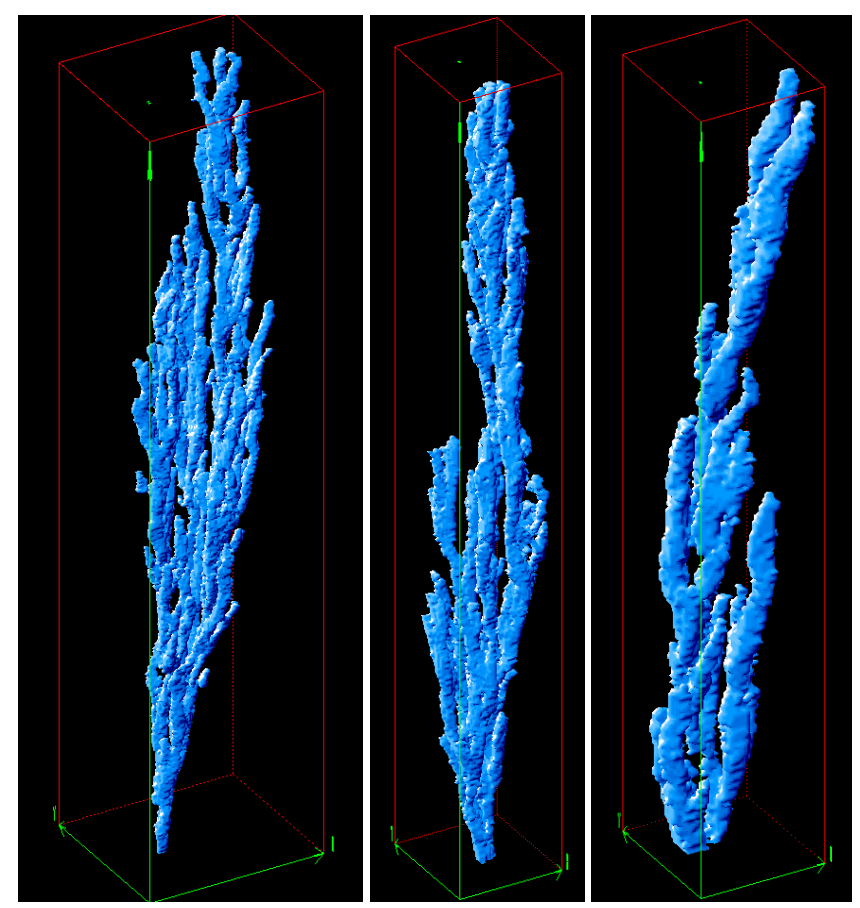

Fig. 10. Three examples of TFGs. From left to right: TFG 15786 lasting $3 \mathrm{~h} 1 \mathrm{~min}$ and extending over 14.6" $\times 13.1^{\prime \prime}$, TFG 1299 ( 2 h $37 \mathrm{~min}$, $\left.6.9^{\prime \prime} \times 7.6^{\prime \prime}\right)$ and TFG 1151 ( $\left.1 \mathrm{~h} 28 \mathrm{~min}, 6.8^{\prime \prime} \times 7.6^{\prime \prime}\right)$.

the scale of the mesogranulation. But for the lower temporal and spatial windows like in Fig. 14 we observe a different behavior like different diverging sources due to close expanding granules of the same TFG. We can say that the repeating fragmenting events of a TFG are equivalent to a positive divergence feature or "a mesogranule", if the temporal window is greater than $30 \mathrm{~min}$.

\section{Relation of granular dynamics to the network}

Following passive scalars, like corks, over long time scales enables the formation of a network at supergranular scale.
This has been done using all the granules to compute the flow field and the location of the corks at the end of the time sequence is shown in Fig. 15. Note that the diffusion coefficient of corks we computed $\left(270 \mathrm{~km}^{2} \mathrm{~s}^{-1}\right)$ is in agreement with previous results (Cadavid et al. 1999). Magnetic fields measured on KPNO magnetograms have been superimposed on the figure, showing a rather good correlation between the cork location and the magnetic network.

In order to correlate the granular dynamics with the network, we plotted in Fig. 16 the density of granule splitting during the whole time sequence along with the final location of the corks. It is striking that corks concentrate at those places and lanes where the density of granule splitting is low; this underlines a close relation between the spatial distribution of the splitting density and the network. We also note that the highest density of splitting is not necessarily located at the centers of the supergranules, some of them being very close to the limit of the network.

Figure 16 also demonstrates clearly that on a long time scale $(9 \mathrm{~h})$ the granule splitting does not occur uniformly over the solar surface - which is a new result. This is related to the existence of long-lived TFGs which concentrate the splitting to a small area. This property is again evident in Fig. 17 where the sum of all large granules (diameter $>1.4^{\prime \prime}$ ), generally associated with the long-lived TFGs, is displayed. The correlation between the splitting density and the large granule density is high, i.e. 0.75 .

\section{Discussion and conclusion}

The visual inspection of a white light granulation movie reveals the extreme complexity of the turbulent convection on the Sun's surface. The difficulties in following visually the complete evolution of solar granules is partly due to the large number of explosions which catch our eyes. We seem to concentrate fully on following them, forgetting the evolution of the granules in the vicinity. To follow automatically the granule evolution step by step, one must identify their temporal links in a 3D space-time box.

The quantitative measure of these links is complicated because granules change shape during their life and it is well known that it is quite difficult to define the life history of a typical granule (Spruit et al. 1990). We described in this paper a method of determining the 3D granule links which takes care of the granule splitting into multiple objects, merging of objects and disappearances or appearances between two successive images. Various tests, performed by varying the parameters of our processing algorithm, showed that our method is quite robust with respect to the temporal labeling of the granules.

The application of this processing to the high spatial resolution sequence (duration $8.75 \mathrm{~h}$ ) observed at the SVST on La Palma in June 1993 (Simon et al. 1994) allowed us to reveal new facets of the properties of the turbulent convection sitting at the top of the convection zone of the Sun. The 3D analysis $(x, y, t)$ of the granulation intensity field demonstrated that a significant fraction of the granules in the photosphere are organized in "Trees of Fragmenting Granules" (TFGs). A TFG consists of a family of repeatedly splitting granules originating 

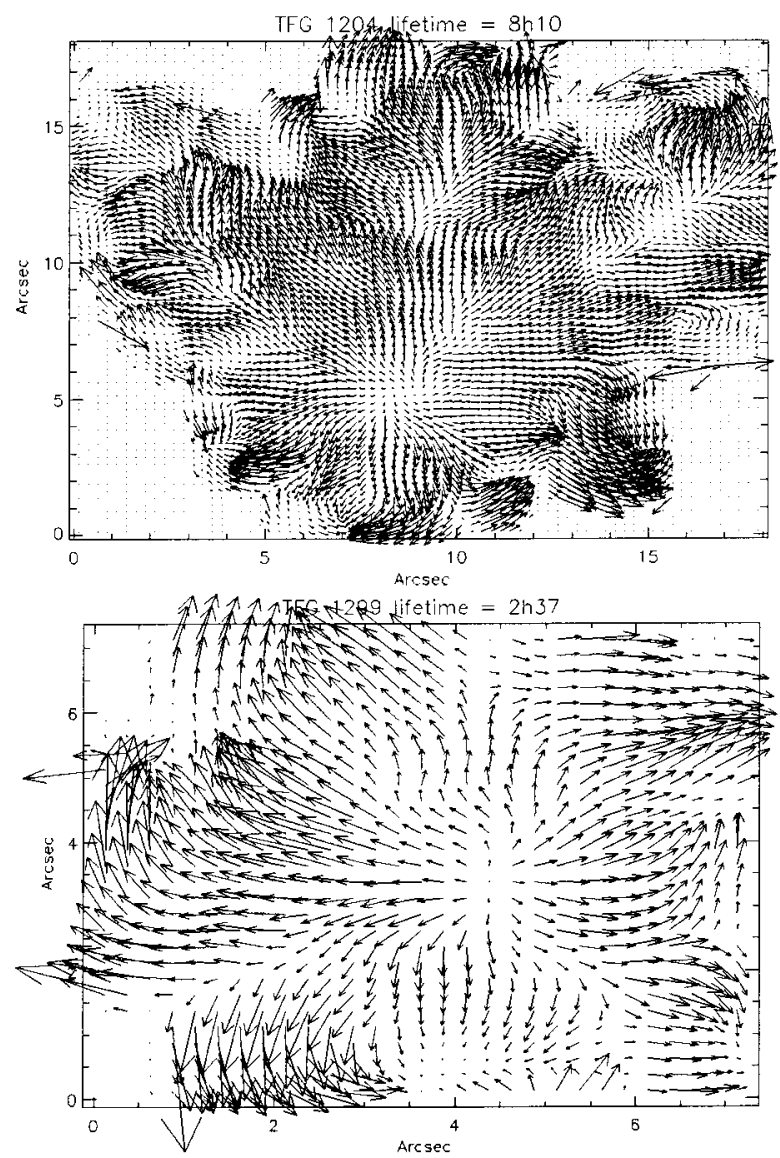
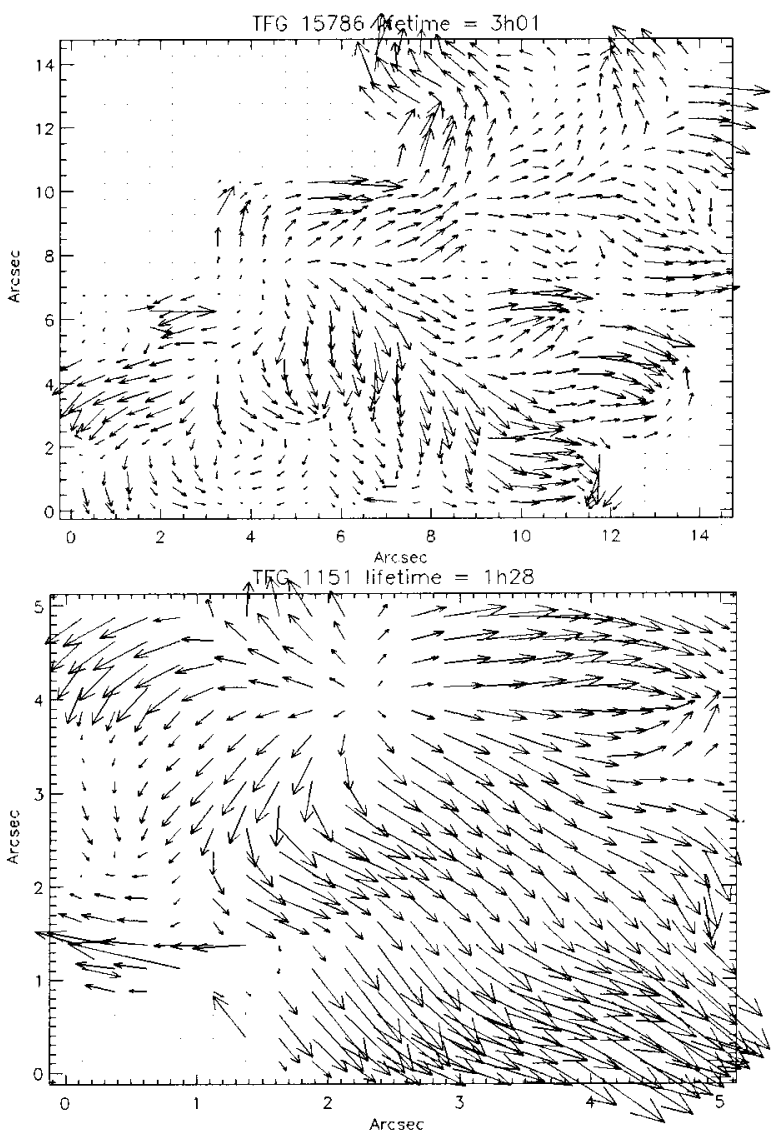

Fig. 11. Four examples of flow fields averaged over the lifetimes of 4 TFGs ( $8 \mathrm{~h} 10 \mathrm{~min}, 3 \mathrm{~h} 1 \mathrm{~min}, 2 \mathrm{~h} 37 \mathrm{~min}, 1 \mathrm{~h} 28 \mathrm{~min}$ ) of our time sequence; they correspond to the longest-lived one shown in Fig. 2 and to the 3 examples of Fig. 10.

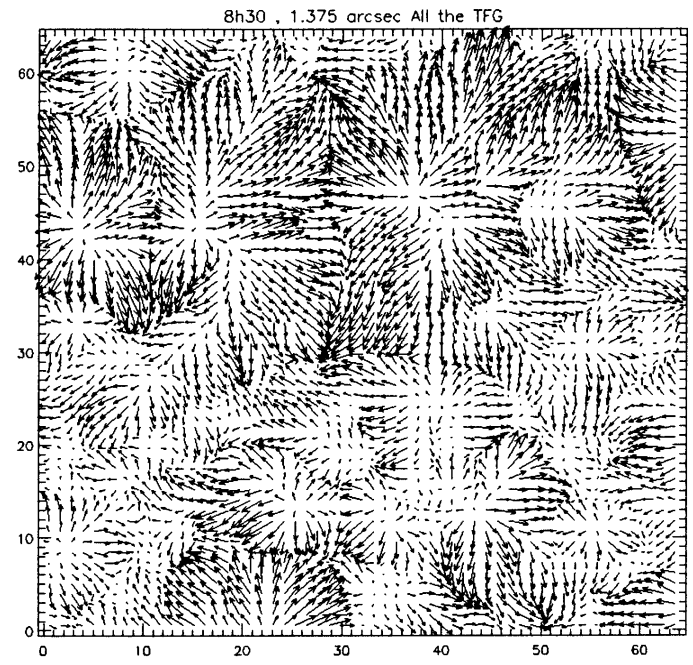

Fig. 12. Flow field averaged over 8 h 45 min.

from a single granule at its beginning. A striking result is that TFGs can live much longer (up to $8 \mathrm{~h} 10 \mathrm{~min}$ ) than individual granules (10 $\mathrm{min})$.

Our study suggests that these longest-lived families are fundamental in structuring the velocity field that we observe on the Sun's surface. First, the longest-lived families $(\geq 1.5 \mathrm{~h})$ cover a large fraction (62\%) of the total granule area (defined

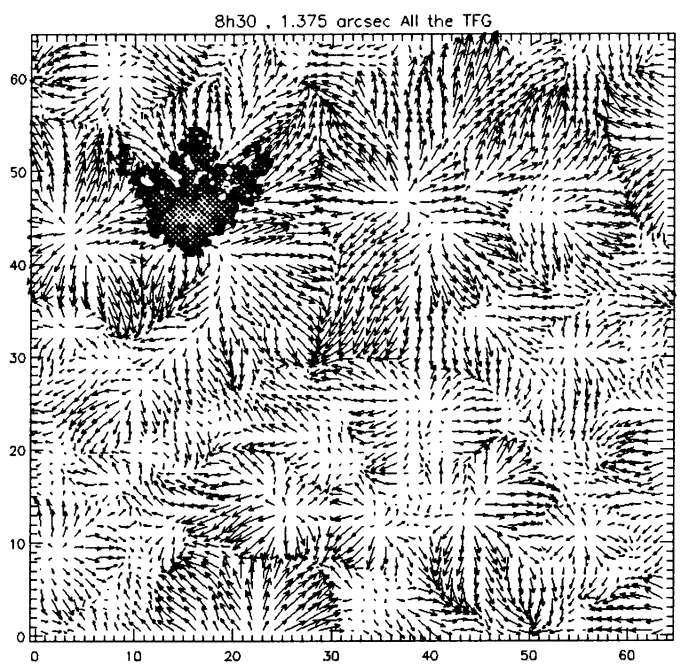

Fig. 13. Flow field averaged over 8 h 45 min with TFG 1204 overplotted.

as per the discussion in Sect. 4). Second, as TFGs lifetimes are much longer than the typical timescale associated with individual granules, the flow induced by such coherent structure should have a strong influence on the dynamics. 

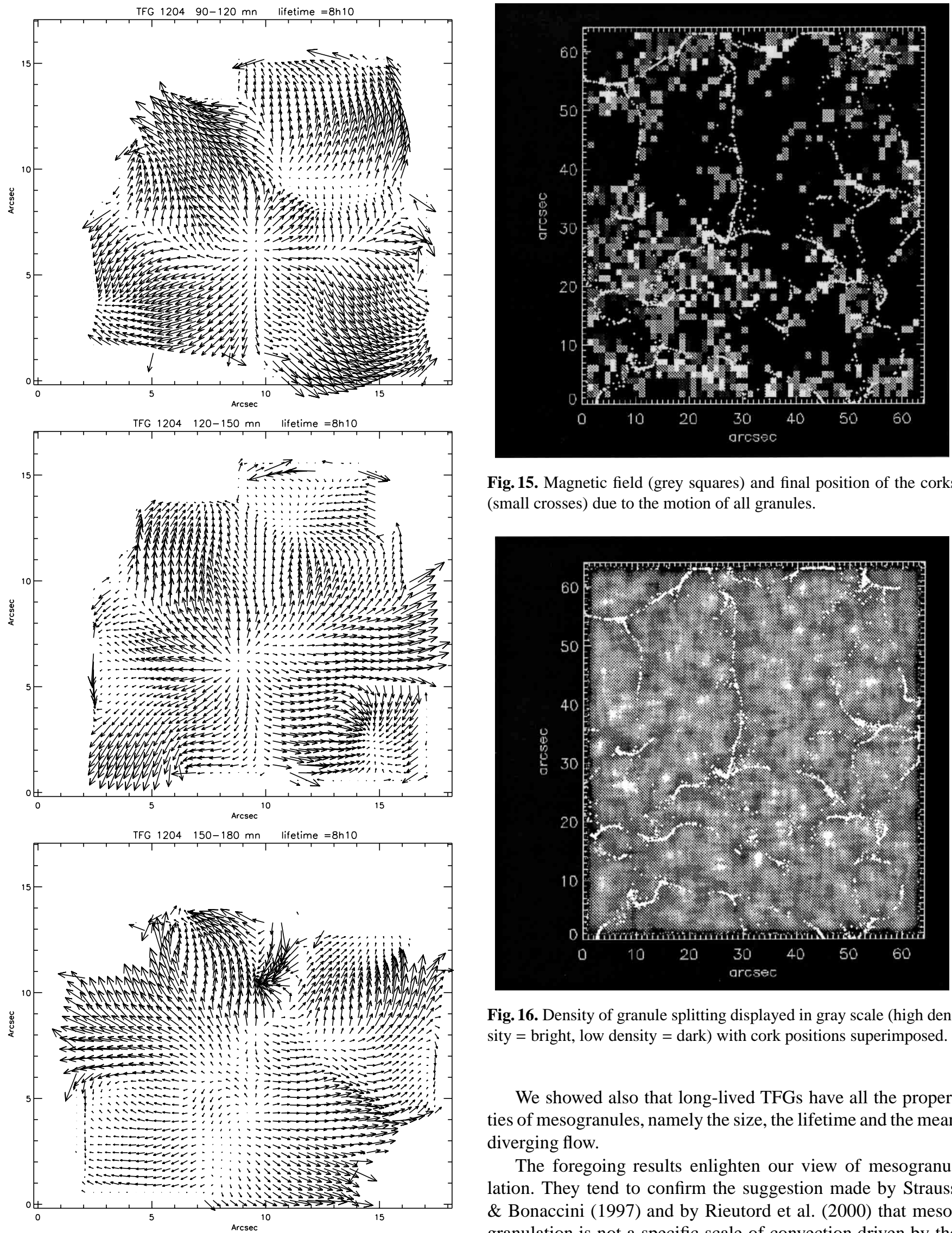

Fig. 15. Magnetic field (grey squares) and final position of the corks (small crosses) due to the motion of all granules.

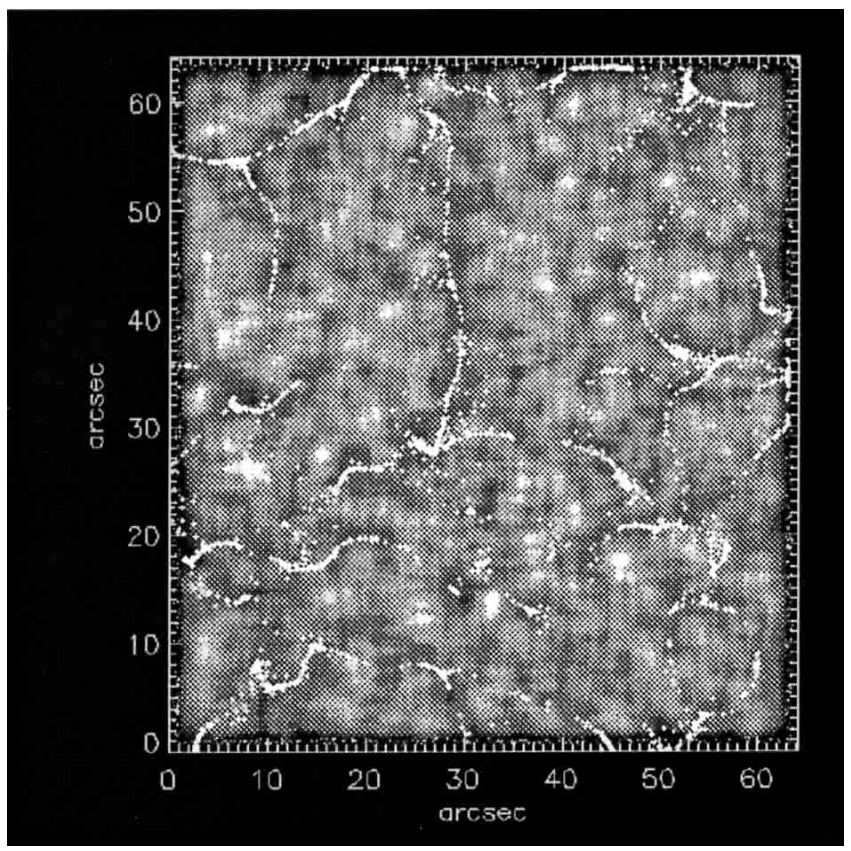

Fig. 16. Density of granule splitting displayed in gray scale (high density $=$ bright, low density $=$ dark) with cork positions superimposed.

We showed also that long-lived TFGs have all the properties of mesogranules, namely the size, the lifetime and the mean diverging flow.

The foregoing results enlighten our view of mesogranulation. They tend to confirm the suggestion made by Strauss \& Bonaccini (1997) and by Rieutord et al. (2000) that mesogranulation is not a specific scale of convection driven by the first ionization of helium, but just the large-scale extension of granulation. TFGs show indeed how a coherent meso-scale structure, a diverging flow, actually builds up from motions at lived TFG (cf. Fig. 2) computed with a temporal window of $30 \mathrm{~min}$ and a spatial window of $1.5^{\prime \prime}$. 


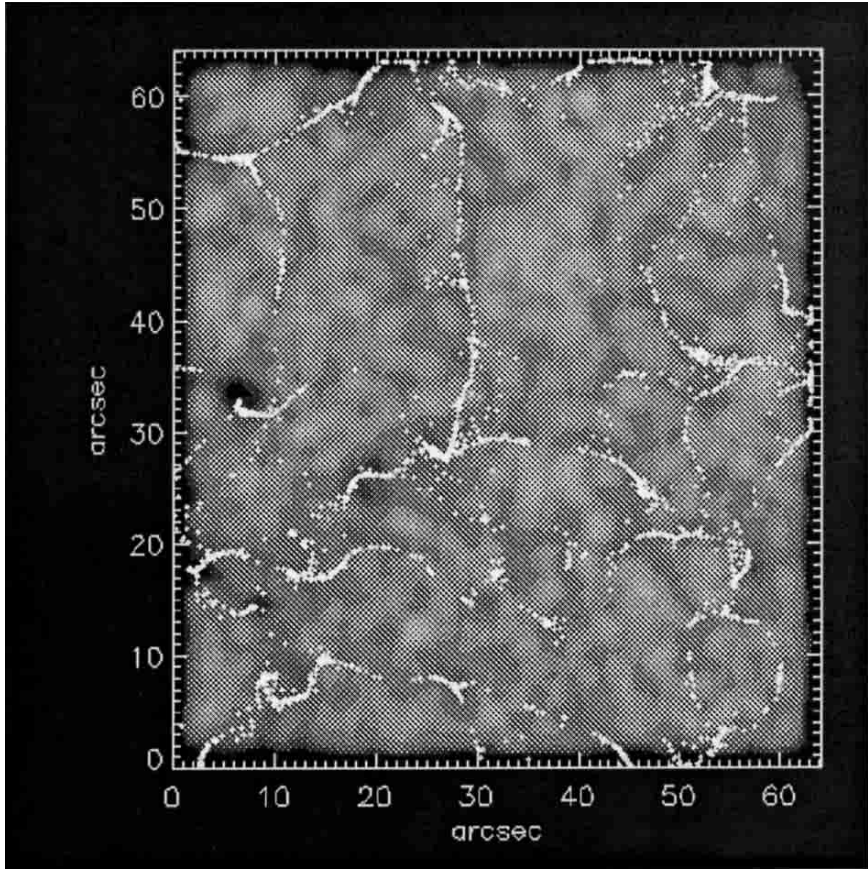

Fig. 17. Positions of corks superimposed on locations of large granules (diameter $>1.4^{\prime \prime}$ ) throughout the whole series, displayed in gray scale (high density $=$ bright, low density $=$ dark).

the granular scale. In this framework, TFGs can be viewed as large scale coherent structures of the solar turbulent convection. Nevertheless the mechanism by which they actually form remains to be understood, as well as the power laws describing their statistical properties. We already note that TFGs are not driven directly by buoyancy since they cover only a fraction of the radiating surface of the Sun.

Note also that these structures, whose maximun radius is around 5", are in fact the real-space side of the spectral feature noticed in Rieutord et al. (2000) when computing the power spectra of horizontal velocities (see their Fig. 1). This spectral feature was associated with "Strong Positive Divergences" by them, but we show here that the true structure is actually a TFG which may contain many "Strong Positive Divergences".

Concerning the relation between TFGs and supergranules, our work shows that the density of splitting granules is very low on the supergranule boundaries. We also found that, on a long time scale $(9 \mathrm{~h})$, the granule splitting does not occur uniformly on the solar surface. This is related to the existence of longlived TFGs which concentrate the splitting within small areas.

In order to investigate in more detail the possible link between TFGs and supergranulation, one should analyze a longer time sequence like the one used by Shine et al. (2000), but with higher spatial and temporal resolution than they used.

The new insight in the organization of the granulation brought by the concept of TFGs could also help to understand other solar surface phenomena. In the following we discuss some of them.

The persistent structures in the averaged continuum images described by Baudin et al. (1997) and Getling \& Brandt (2002) can be interpreted as a consequence of the TFGs which concentrate granules in certain areas in the space-time box.
Hoekzema \& Brandt (2000) pointed out the importance of meso-scale flows for solar surface waves. We think that TFGs may play a role in the excitation of these oscillations. Indeed, in the TFGs the splitting granules (or exploders) originating from the same parent evolve nearly parallel in time and at close locations. They split more or less in phase and this introduces an instantaneous large coherent scale. This scale is larger than that of the granulation for the strong downflow where the excitation of the oscillations occurs preferentially (Rimmele et al. 1995). This is in agreement with previous results (Roudier et al. 1997) where holes form along the dark lane (downflow) continuously visible for more than $45 \mathrm{~min}$ and systematically distributed at the periphery of mesogranular cells (i.e. the TFGs).

According to November (1989) the mesogranulation is the principal component of the vertical velocity for the supergranular flow. If we identify TFGs with mesogranules, this indicates the important contribution of the TFGs in the supergranular flows. The cork evolution shows that all families contribute to form the network but the effect of the long-lived ones alone $(>1.5 \mathrm{~h}$ ) could be sufficient to produce the network. However, the motions could be influenced by an existing largescale flow which we cannot exclude. More investigations by numerical simulations are needed to find out if the action of the cumulative effects of the TFGs alone are sufficient to create the network.

The treatment of the anomalous transport in disordered media of magnetic elements (Cadavid et al. 1999) invokes, to interpret their diffusion measurements, "a memory"of the underlying convection. Such a behaviour of the magnetic field diffusion could be ascribed now to the action of TFGs. Indeed, TFGs last up to 8 hours which could represent the sought-after aspect of "memory" required for the granular pattern.

Our findings concerning TFGs and in particular the power laws characterizing their statistical properties should serve as a reference for numerical simulations of the solar surface convection. The existence and properties of TFGs in the large scale numerical simulation described in Rieutord et al. (2001) are currently being investigated.

Acknowledgements. This work was supported by the Centre National de la Recherche Scientifique (CNRS, UMR 5572) and the Programme National Soleil Terre (P.N.S.T.). The authors thank Kitt Peak Observatory (USA) for providing the magnetograms. The support by G. Hosinsky, R. Kever, G. Scharmer, G. W. Simon, and W. Wang in taking the observations as well as by W. Pötzi in the pre-reduction of the data is gratefully acknowledged. The SVST is operated on the island of La Palma by the Royal Swedish Academy of Sciences at the Spanish Observatorio del Roque de los Muchachos of the IAC.

\section{References}

Baudin, F., Molowny-Horas, R., \& Koutchmy, S. 1997, A\&A, 326, 842

Cadavid, A. C., Lawrence, J. K., \& Ruzmaikin, A. 1999, ApJ, 521, 844

Getling, A. V., \& Brandt, P. N. 2002, A\&A, 382, L5

Hagenaar, H. J., Schrijver, C. J., \& Title, A. M. 2003, ApJ, 584, 1107

Harvey, J. W. 1965, PASP, 77, 129 
Hoekzema, N. M., \& Brandt, P. N. 2000, A\&A, 353, 389

Kawaguchi, I. 1980, Solar Phys., 65, 207

Muller, R., Auffret, H., Roudier, Th., et al. 1992, Nature, 356, 322

Müller, D. A. N., Steiner, O., Schlichenmaier, R., \& Brandt, P. N. 2001, Sol. Phys., 203, 211

November, L. J. 1989, ApJ, 344, 494

Ploner, S. R. O. 1998, Ph.D. Thesis (Zürich)

Rieutord, M., Roudier, T., Malherbe, J. M., \& Rincon, F. 2000, A\&A, 357, 1063

Rieutord, M., Roudier, T., Ludwig, H. G., Nordlund, Å., \& Stein, R. 2001, A\&A, 377, 14

Rimmele, T .R., Goode, P. R., Harold, E., \& Stebbins, R. T. 1995, ApJ, 444,119
Roudier, T., Malherbe, J. M., November, L., et al. 1997, A\&A, 320, 605

Shine, R. A., Simon, G. W., \& Hurlburt, N. E. 2000, Solar Phys., 193, 313

Simon, G. W., Title, A. M., \& Weiss, N. O. 1991, ApJ, 375, 775

Simon, G. W., Brandt, P. N., November, L. J., et al. 1994, Large-scale photospheric motions: first results from an extraordinary elevenhour granulation observation, in Solar Surface Magnetism, ed. R. J. Rutten, \& C. J. Schrijver (Dordrecht: Kluwer), 261

Spruit, H. C., Nordlund, A., \& Title, A. M. 1990, ARA\&A, 28, 263

Straus, T., \& Bonaccini, D. 1997, A\&A, 324, 704

Strous, L. H. 1994, Ph.D. Thesis, Utrecht

Title, A. M., Tarbell, T. D., Topka, K. P., et al. 1989, ApJ, 336, 475 